Research Article

\title{
Combination of Exercise Training and SOD Mimetic Tempol Enhances Upregulation of Nitric Oxide Synthase in the Kidney of Spontaneously Hypertensive Rats
}

\author{
Pengyu Cao (iD, ${ }^{1,2}$ Osamu Ito, ${ }^{2}$ Daisuke Ito, ${ }^{2}$ Rong Rong, ${ }^{2}$ Yang Zheng, \\ and Masahiro Kohzuki ${ }^{2}$ \\ ${ }^{1}$ Cardiovascular Disease Center, The First Hospital of Jilin University, Changchun, China \\ ${ }^{2}$ Department of Internal Medicine and Rehabilitation Science, Tohoku University Graduate School of Medicine, Sendai, Japan \\ Correspondence should be addressed to Pengyu Cao; caopy1979@outlook.com
}

Received 13 March 2020; Revised 12 August 2020; Accepted 30 September 2020; Published 21 October 2020

Academic Editor: Salvatore Corrao

Copyright (c) 2020 Pengyu Cao et al. This is an open access article distributed under the Creative Commons Attribution License, which permits unrestricted use, distribution, and reproduction in any medium, provided the original work is properly cited.

\begin{abstract}
Both exercise training (Ex) and superoxide dismutase (SOD) mimetic tempol have antihypertensive and renal protective effects in rodent models of several hypertensions. We recently reported that Ex increases nitric oxide (NO) production and the expression levels of endothelial and neuronal NO synthase (eNOS and nNOS) in the kidney and aorta of the spontaneously hypertensive rats (SHR) and normotensive Wistar-Kyoto rats (WKY). We also found that endogenous hydrogen peroxide $\left(\mathrm{H}_{2} \mathrm{O}_{2}\right)$ upregulates the expression levels of eNOS and nNOS in SHR. To elucidate the mechanism of the Ex-upregulated NO system in the kidney, we examined the additive effect of Ex and tempol on the renal NO system in SHR and WKY. Our data showed that, in SHR, both Ex and tempol increase the levels of $\mathrm{H}_{2} \mathrm{O}_{2}$ and nitrate/nitrite (NOx) in plasma and urine. We also observed an increased renal NOS activity and upregulated expression levels of eNOS and nNOS with decreased NADPH oxidase activity. The effects of the combination of Ex and tempol on these variables were cumulate in SHR. On the other hand, we found that Ex increases these variables with increased renal NADPH oxidase activity, but tempol did not change these variables or affect the Ex-induced upregulation in the activity and expression of NOS in WKY. The SOD activity in the kidney and aorta was activated by tempol only in SHR, but not in WKY; whereas Ex increased SOD activity only in the aorta in both SHR and WKY. These results indicate that Ex-induced endogenous $\mathrm{H}_{2} \mathrm{O}_{2}$ produced in the blood vessel and other organs outside of the kidney may be carried to the kidney by blood flow and stimulates the NO system in the kidney.
\end{abstract}

\section{Introduction}

Exercise training (Ex) reduces the systemic blood pressure in human [1] and the hypertensive animal model including spontaneously hypertensive rats (SHR) [2, 3], Dahl saltsensitive rats [4], and angiotensin II-infused rats [5]. In addition to antihypertensive effects, Ex offers renal protective effects through decreasing plasma creatinine and proteinuria and improving glomerular sclerosis, in rats with chronic renal failure $[6,7]$ or diabetic nephropathy [8]. However, the underlying mechanisms have not been fully understood.

Nitric oxide (NO) is a vasodilatory factor that can be synthesized by three isoforms of NO synthase (NOS): endothelial, neuronal, and inducible NOS (eNOS, nNOS, and iNOS), and it controls the systemic blood pressure and peripheral hemodynamics [9]. NO has various renal effects including the regulation of renal hemodynamics and the inhibition of renin secretion, tubular $\mathrm{Na}$ reabsorption, tubuloglomerular feedback, and sympathetic nerve activity $[10,11]$. It has been reported that Ex increases the blood flow and NO production in the heart, aorta, and skeletal muscle $[12,13]$. We recently reported that Ex increased NOS activity and expression levels of eNOS and nNOS in the aorta and kidney of SHR and normotensive Wistar-Kyoto rats (WKY) [14].

Superoxide anion $\left(\mathrm{O}_{2}^{-}\right)$is generated by NADPH oxidase $[15,16]$ and metabolized into hydrogen peroxide $\left(\mathrm{H}_{2} \mathrm{O}_{2}\right)$ by 
superoxide dismutase (SOD). Reactive oxygen species (ROS) and oxidative stress are elevated in SHR [17, 18] due to increased NADPH oxidase activity [19-21] and decreased SOD activity [22]. The SOD mimetic tempol lowers blood pressure and ameliorates the impaired vasodilatory responses in SHR $[23,24]$. The expression levels of eNOS and nNOS and the NO production are elevated in the kidney and vessels of SHR compared to WKY [25-27]. In this regard, we recently reported that tempol further increases the mRNA levels of eNOS and nNOS in the kidney and aorta of SHR, and that $\mathrm{H}_{2} \mathrm{O}_{2}$ administered intravenously increases the levels of eNOS and nNOS in the kidney and aorta of WKY [27], suggesting that $\mathrm{H}_{2} \mathrm{O}_{2}$ could mediate the expression of NOS in the kidney and vessels of SHR. $\mathrm{H}_{2} \mathrm{O}_{2}$ may increase NOS expression and activity by improving the rate of gene transcription and mRNA processing and stability [28] and increasing the levels of phosphorylated eNOS at Ser1177 [27].

Previous studies reported that Ex increases the extracellular SOD (ecSOD) and eNOS expression in the mouse aorta [29], and the Ex-induced eNOS is mediated through $\mathrm{H}_{2} \mathrm{O}_{2}$ in the aorta of mice [30]. Considering that shear stress [31] and $\mathrm{H}_{2} \mathrm{O}_{2}$ [28] increases the expression of eNOS in cultured bovine aortic endothelial cells, we hypothesize that tempol enhances the effect of Ex on the NO system in the kidney of SHR. In the present study, we sought to clarify the mechanism of the Ex-upregulated NO system in the kidney through examining the additive effect of Ex and tempol on the renal NO system and oxidative stress in SHR and WKY.

\section{Methods}

2.1. Animal and Experimental Protocol. Five-week-old male SHR/Izm and WKY/Izm were obtained from SLC (Shizuoka, Japan). These rats were housed in the animal care facility at the Tohoku University School of Medicine under controlled temperature $\left(24^{\circ} \mathrm{C}\right)$ and a $12 \mathrm{~h}$ light-dark cycle. All rats had free access to standard laboratory chow and water. All protocols involving rats were reviewed and received prior approval by the Animal Welfare Committee at the Tohoku University School of Medicine.

SHR and WKY were randomly divided into four groups ( $n=10$ in each group): control, Ex, tempol-treated (Tmp), and an Ex + Tmp. The treadmill running $(20 \mathrm{~m} / \mathrm{min}, 60 \mathrm{~min} /$ day, and 6 times/week) was performed with a rat treadmill (KN-73, Natsume Industries Co., Tokyo, Japan) to the Ex and Ex + Tmp groups, and tempol $(1 \mathrm{mmol} / \mathrm{l})$ was given to the Tmp and Ex + Tmp groups through drinking water for 8 weeks.

\subsection{Blood Pressure Monitoring and Preparation of Plasma and} Urinary Samples. The systolic blood pressure (SBP) was monitored by the tail-cuff method (Model UR-5000, Ueda, Tokyo, Japan). The rats were placed metabolic cages (Model ST; Sugiyama-General, Tokyo, Japan) individually on the day before the final experimental day, and urine samples were collected on ice over a period of $24 \mathrm{~h}$. On the final experimental day, the rats were anesthetized with pentobarbital sodium (50 mg/kg, i.p.), and blood samples were collected by decapitation. These samples were centrifuged for 5 minutes at $1,500 \mathrm{rpm}$ and separated from the sediments and stored at $-80^{\circ} \mathrm{C}$.

\subsection{Plasma and Urinary Biochemical Variables Measurement.} Creatinine and urea nitrogen were measured by a standard autoanalysis technique, (BML, Tokyo, Japan). $\mathrm{H}_{2} \mathrm{O}_{2}$ was measured using an Amplex Red Hydrogen Peroxide/Peroxidase Assay kit (Molecular Probes, OR, USA) [32]. Nitrate/nitrite (NOx) was measured spectrophotometrically by the Griess reagent method [33] using the Nitrate/Nitrite Colorimetric Assay Kit (Cayman Chemical Company, MI, USA).

2.4. Measurement of NADPH Oxidase, SOD, and NOS Activities. The preparation of tissue samples including the renal cortex (CO), outer medulla (OM), inner medulla (IM), and aorta was described previously [27]. The protein concentration of the samples was determined using the Bradford method [34]. The NADPH oxidase activity was assessed as an index of $\mathrm{O}_{2}^{-}$generation by the lucigenin-enhanced chemiluminescence method [14,27] and presented as counts per minute $(\mathrm{CPM}) / \mathrm{mg}$ of protein. The NADPH oxidase activity was examined in the CO, OM, and aorta, as it is undetectable in the IM. The SOD activity was measured by the Superoxide Dismutase Assay Kit (Cayman Chemical Company, MI, USA) utilizing a tetrazolium salt for detection of superoxide radicals generated by xanthine oxidase and hypoxathine. One unit (U) of SOD is defined as the amount of enzyme needed to exhibit 50\% dismutation of the superoxidase radical. SOD activity was presented as $\mathrm{U} / \mathrm{g}$ tissue protein. For the determination of NOS activity, the in vitro formation of $\mathrm{NOx}$ by each tissue was evaluated by using a colorimetric NOS activity assay kit (Oxford Biomedical Research, Inc., Rochester Hills, MI, USA) as described previously [14]. The NO production determined by NOx formation was linear with time and protein concentration. Data were presented as $\mu \mathrm{mol} / \mathrm{g}$ protein/unit time for the NOS activity.

2.5. Immunoblot. The level of NOS was examined by Western blot, as described previously $[27,35]$. The relative intensities of the bands at the $140 \mathrm{kDa}$ for eNOS, $155 \mathrm{kDa}$ for nNOS, and $130 \mathrm{kDa}$ for iNOS were quantified using the Image J software (version 1.40, National Institutes of Health, $\mathrm{MD}$, USA) and normalized to $\beta$-actin.

2.6. Statistical Analysis. Data are presented as the mean$\mathrm{s} \pm \mathrm{SEM}$. The comparison between the WKY control and SHR control used the one-way ANOVA. In WKY or SHR, compared with the control group, Ex, Tmp, and Tmp + Ex were all equivalent to the single factor intervention group, so one-way ANOVA was used. Subsequently, for the comparison between Ex, Tmp, and Tmp + Ex groups, data analysis was performed by Fisher's probability least significant difference (LSD) test for multiple comparisons after 
one-way ANOVA. In all analyses, a two-tailed $P<0.05$ was considered as statistical significance. All statistical analysis data were performed using the SPSS 19 software (IBM Corp., Armonk, NY, USA).

\section{Results}

3.1. Effects of Ex and Tempol on Blood Pressure and Biochemical Variables in Plasma and Urine. The SBP, DBP, body weight, kidney weight, and plasma variables in the four groups of SHR and WKY are shown in Tables 1-3. The SBP, $\mathrm{DBP}$, triglyceride, glucose, renal and aorta NADPH oxidase activities, and plasma and urinary $\mathrm{H}_{2} \mathrm{O}_{2}$ and $\mathrm{NOx}$ were significantly higher in the control SHR group than in the control WKY group $(P<0.01)$, but plasma creatinine and urea nitrogen and creatinine clearance were not significantly different between these control groups. In SHR, Ex and tempol significantly decreased the SBP, and there is an additive effect of the combination of Ex and tempol on the SBP. Ex significantly decreased the plasma creatinine and increased creatinine clearance (Ccr), which reflects the glomerular filtration rate (GFR), and tempol tends to increase $\operatorname{Ccr}(P=0.062)$. Other variables were not different among the four groups. In contrast to SHR, neither Ex nor tempol affected SBP or plasma variables in WKY.

In SHR, Ex and tempol significantly increased the levels of $\mathrm{H}_{2} \mathrm{O}_{2}$ in plasma and urine (Ex: by $21 \%$ and $26 \%, P<0.01$; tempol: by $23 \%$ and $24 \%, P<0.01)$ compared to controls. Notably, the combination of Ex and tempol further increased $\mathrm{H}_{2} \mathrm{O}_{2}$ in plasma and urine by $50 \%$ and $62 \%(P<0.01)$ compared to the control group, and the differences between the Ex + Tmp and the Ex and Tmp only groups reached statistical significance $(P<0.01$, respectively) (Figure 1$)$. In WKY, Ex increased the level of $\mathrm{H}_{2} \mathrm{O}_{2}$ by $36 \%$ in urine $(P<0.05)$ compared to controls, but Tmp did not significantly change it. The combination of Ex and Tmp increased $\mathrm{H}_{2} \mathrm{O}_{2}$ by $35 \%$ in urine $(P<0.05)$ compared to controls, but the plasma and urinary $\mathrm{H}_{2} \mathrm{O}_{2}$ levels were not significantly different between the $\mathrm{Ex}+\mathrm{Tmp}$ group and Ex only group (Figure 1).

In SHR, Ex and Tmp significantly increased the NOx in plasma and urine, respectively (Ex: by $19 \%$ and $22 \%, P<0.01$; tempol: by $18 \%$ and $26 \% P<0.01)$; the combination of Ex and Tmp further increased the NOx in plasma and urine to $38 \%$ and $47 \%$ compared to the controls $(P<0.01)$, and the NOx levels in the plasma and urinary were significantly higher in the Ex + Tmp group compared to the Ex and tempol groups $(P<0.01)$ (Figure 2). In WKY, Ex significantly increased the NOx in plasma and urine by $22 \%$ and $37 \%$, but Tmp did not change them; the combination of Ex and Tmp increased the NOx in plasma and urine by $26 \%$ and $43 \%$ compared to controls $(P<0.01)$, but they were not significantly different in the Ex + Tmp group compared to the Ex group (Figure 2).

3.2. Effects of Ex and Tmp on NADPH Oxidase and SOD Activities. In SHR, Ex and Tmp significantly decreased the activity of NADPH oxidase in the CO, OM, and aorta. An additive effect of the combination of Ex and Tmp on the NADPH oxidase activity in these tissues was also found
(Figure 3(a)). In WKY, Ex significantly increased the NADPH oxidase activity in the CO, OM, and aorta, but Tmp did not change the NADPH oxidase activity in these tissues; the combination of Ex and Tmp significantly increased the NADPH oxidase activity in the CO, OM, and aorta compared to controls, but not the Ex only group (Figure 3(b)).

In SHR, Ex significantly increased SOD activity in the IM and aorta, but not in the CO and OM; Tmp significantly increased SOD activity in the kidney sections and aorta; there is an additive effect of the combination of Ex and Tmp on the SOD activity in the IM and aorta (Figure 4(a)). In WKY, Ex significantly increased SOD activity in the aorta, but not in the kidney sections; whereas Tmp did not change SOD activity in these tissues, and the combination of Ex and Tmp did not further increase SOD activity compared to the Ex only group (Figure 4(b)).

3.3. Effects of Ex and Tmp on the Level and Activity of NOS. In SHR, Ex and Tmp significantly increased NOS activity in the kidney sections and aorta; an additive effect on NOS activity was observed for Ex and Tmp in the kidney sections and aorta (Figure 5(a)). In WKY, Ex significantly increased NOS activity in the kidney sections and aorta, but Tmp did not change the activity in these tissues; the combination of Ex and tempol increased NOS activity in the kidney sections and aorta compared to the controls, but not to the Ex group (Figure 5(b)).

The protein levels of eNOS and nNOS in the kidney sections and aorta were also examined. As shown in Figure 6, in SHR, Ex and Tmp significantly increased the level of eNOS in the kidney sections and aorta; and an additive effect of Ex and Tmp on the level of eNOS was found in the kidney sections and aorta (Figure 6(a)). In WKY, Ex significantly increased the protein level of eNOS in the kidney sections and aorta, but Tmp did not change it in these tissues. The combination of Ex and Tmp increased the eNOS protein level in the kidney sections and aorta compared to the controls, but not the Ex group (Figure 6(b)).

As shown in Figure 7, in SHR, Ex and Tmp significantly increased the level of nNOS in the kidney sections and aorta; and there is an additive effect of Ex and Tmp on the protein level of nNOS in the kidney sections and aorta (Figure 7(a)). In WKY, Ex significantly increased the protein level of nNOS in the kidney sections and aorta, but Tmp did not in these tissues. The combination of Ex and Tmp increased the protein level of nNOS in the kidney sections and aorta compared to the controls, but not the Ex group (Figure 7(b)). Neither Ex or Tmp affected the iNOS level in the kidney sections and aorta of both strains of rats (data not shown).

\section{Discussion}

Although Ex increased the NOS activity and protein levels of eNOS and nNOS in the aorta and kidney of SHR and WKY, the mechanism of the Ex-upregulated NO system in the kidney has not yet been discovered. In the present study, we found that Ex increases concomitantly plasma and urinary $\mathrm{H}_{2} \mathrm{O}_{2}$ and $\mathrm{NOx}$ levels and renal NOS expression with decreased NADPH oxidase activity in SHR, but with increased NADPH oxidase 
TABLE 1: The SBP and biochemical variables in SHR control and WKY control.

\begin{tabular}{|c|c|c|}
\hline & $\begin{array}{c}\text { WKY } \\
\text { Control }(n=10)\end{array}$ & $\begin{array}{c}\text { SHR } \\
\text { Control }(n=10)\end{array}$ \\
\hline SBP (mmHg) & $153 \pm 8$ & $225 \pm 6^{* *}$ \\
\hline DBP (mmHg) & $105 \pm 4$ & $130 \pm 5^{* *}$ \\
\hline Body weight (g) & $378 \pm 16$ & $386 \pm 15$ \\
\hline Kidney weight/body weight $(\mathrm{g} / \mathrm{kg})$ & $6.21 \pm 0.53$ & $6.48 \pm 0.27$ \\
\hline Total cholesterol (mg/dl) & $69 \pm 4.55$ & $64.40 \pm 2.08$ \\
\hline Triglyceride $(\mathrm{mg} / \mathrm{dl})$ & $32.17 \pm 3.27$ & $53.00 \pm 4.39^{* *}$ \\
\hline Free fatty acid $(\mathrm{mEq} / \mathrm{l})$ & $0.24 \pm 0.02$ & $0.21 \pm 0.01$ \\
\hline Glucose (mg/dl) & $177.17 \pm 5.36$ & $199.80 \pm 5.62^{* *}$ \\
\hline Plasma $\mathrm{H}_{2} \mathrm{O}_{2}(\mathrm{umol} / \mathrm{L})$ & $1.71 \pm 0.22$ & $3.51 \pm 0.18^{* *}$ \\
\hline Urine $\mathrm{H}_{2} \mathrm{O}_{2}$ (nmol/day) & $19.73 \pm 1.46$ & $26.45 \pm 1.24^{*}$ \\
\hline Plasma NOx (umol/L) & $9.78 \pm 0.39$ & $16.55 \pm 0.39^{* *}$ \\
\hline Urine NOx (umol/day) & $0.30 \pm 0.03$ & $0.53 \pm 0.03^{* *}$ \\
\hline Renal cotex NADPH oxidase activity (counts/min/mg) & $6624 \pm 388$ & $27743 \pm 687^{* *}$ \\
\hline Renal outer medulla NADPH oxidase activity (counts/min/mg) & $6362 \pm 368$ & $21038 \pm 625^{* *}$ \\
\hline Aorta NADPH oxidase activity (counts/min/mg) & $13352 \pm 487$ & $43644 \pm 866^{* *}$ \\
\hline Plasma creatinine $(\mathrm{mg} / \mathrm{dl})$ & $0.19 \pm 0.01$ & $0.17 \pm 0.01$ \\
\hline Creatinine clearance $(\mathrm{ml} / \mathrm{min})$ & $2.15 \pm 0.31$ & $2.50 \pm 0.51$ \\
\hline Plasma urea nitrogen $(\mathrm{mg} / \mathrm{dl})$ & $18.88 \pm 0.63$ & $17.86 \pm 0.60$ \\
\hline
\end{tabular}

Values are means \pm SEM. ${ }^{*} P<0.05$ vs. the WKY control group. ${ }^{* *} P<0.01$ vs. the WKY control group.

TABLE 2: Effects of Ex and tempol on the SBP and biochemical variables in SHR.

\begin{tabular}{|c|c|c|c|c|}
\hline & Control $(n=10)$ & $\operatorname{Ex}(n=10)$ & $\operatorname{Tmp}(n=10)$ & $\mathrm{Ex}+\operatorname{Tmp}(n=10)$ \\
\hline SBP (mmHg) & $225 \pm 6$ & $211 \pm 4^{* *}$ & $212 \pm 6^{* *}$ & $202 \pm 4^{* * \#+}$ \\
\hline DBP (mmHg) & $130 \pm 5$ & $122 \pm 5$ & $125 \pm 3$ & $123 \pm 3$ \\
\hline Body weight (g) & $386 \pm 15$ & $350 \pm 23$ & $366 \pm 13$ & $358 \pm 19$ \\
\hline Kidney weight/body weight $(\mathrm{g} / \mathrm{kg})$ & $6.48 \pm 0.27$ & $5.93 \pm 0.19$ & $6.04 \pm 0.29$ & $6.12 \pm 0.11$ \\
\hline Total cholesterol (mg/dl) & $64.40 \pm 2.08$ & $61.80 \pm 2.35$ & $59.20 \pm 2.11$ & $59.00 \pm 2.25$ \\
\hline Triglyceride $(\mathrm{mg} / \mathrm{dl})$ & $53.00 \pm 4.39$ & $61.40 \pm 4.66$ & $51.80 \pm 5.75$ & $61.20 \pm 5.04$ \\
\hline Free fatty acid $(\mathrm{mEq} / \mathrm{l})$ & $0.21 \pm 0.01$ & $0.20 \pm 0.02$ & $0.20 \pm 0.02$ & $0.18 \pm 0.02$ \\
\hline Glucose $(\mathrm{mg} / \mathrm{dl})$ & $199.80 \pm 5.62$ & $187.80 \pm 5.85$ & $187.00 \pm 7.50$ & $193.40 \pm 4.12$ \\
\hline Plasma creatinine $(\mathrm{mg} / \mathrm{dl})$ & $0.17 \pm 0.01$ & $0.11 \pm 0.02^{* *}$ & $0.14 \pm 0.02$ & $0.08 \pm 0.01^{* *+}$ \\
\hline Creatinine clearance $(\mathrm{ml} / \mathrm{min})$ & $2.50 \pm 0.51$ & $4.57 \pm 0.67^{* *}$ & $3.28 \pm 0.46$ & $5.82 \pm 0.75^{* *+}$ \\
\hline Plasma urea nitrogen $(\mathrm{mg} / \mathrm{dl})$ & $17.86 \pm 0.60$ & $17.34 \pm 0.75$ & $17.28 \pm 0.73$ & $16.78 \pm 0.79$ \\
\hline
\end{tabular}

Values are means \pm SEM. ${ }^{*} P<0.05$ vs. the control group. ${ }^{* *} P<0.01$ vs. the control group. ${ }^{\#} P<0.05$ vs. the Ex group. ${ }^{+} P<0.05$ vs. the Tmp group.

TABLE 3: Effects of Ex and tempol on the SBP and biochemical variables in WKY.

\begin{tabular}{lcccc}
\hline & Control $(n=10)$ & Ex $(n=10)$ & Tmp $(n=10)$ & Ex + Tmp $(n=10)$ \\
\hline SBP $(\mathrm{mmHg})$ & $153 \pm 8$ & $155 \pm 5$ & $153 \pm 6$ & $154 \pm 6$ \\
DBP (mmHg) & $105 \pm 4$ & $108 \pm 3$ & $102 \pm 4$ & $103 \pm 3$ \\
Body weight (g) & $378 \pm 16$ & $359 \pm 22$ & $362 \pm 12$ & $356 \pm 19$ \\
Kidney weight/body weight $(\mathrm{g} / \mathrm{kg})$ & $6.21 \pm 0.53$ & $6.34 \pm 0.33$ & $6.03 \pm 0.26$ & $6.28 \pm 0.17$ \\
Total cholesterol (mg/dl) & $69 \pm 4.55$ & $69.80 \pm 4.35$ & $65.50 \pm 4.39$ & $64.60 \pm 4.11$ \\
Triglyceride (mg/dl) & $32.17 \pm 3.27$ & $37.80 \pm 5.66$ & $31.80 \pm 5.75$ & $34.20 \pm 5.04$ \\
Free fatty acid (mEq/l) & $0.24 \pm 0.02$ & $0.26 \pm 0.02$ & $0.26 \pm 0.02$ & $0.21 \pm 0.02$ \\
Glucose (mg/dl) & $177.17 \pm 5.36$ & $167.60 \pm 7.03$ & $179.80 \pm 6.93$ & $165.40 \pm 7.12$ \\
Plasma creatinine (mg/dl) & $0.19 \pm 0.01$ & $0.17 \pm 0.01$ & $0.18 \pm 0.01$ & $0.16 \pm 0.01$ \\
Creatinine clearance (ml/min) & $2.15 \pm 0.31$ & $2.45 \pm 0.41$ & $2.29 \pm 0.35$ & $2.64 \pm 0.42$ \\
Plasma urea nitrogen (mg/d) & $18.88 \pm 0.63$ & $17.36 \pm 0.69$ & $20.75 \pm 0.84$ & $17.38 \pm 0.78$ \\
\hline
\end{tabular}

activity in WKY. We also observed an additive effect of Tmp and Ex on these variables in SHR, but not in WKY.

In our previous study, we found that Tmp increases the plasma and urinary $\mathrm{H}_{2} \mathrm{O}_{2}$ and $\mathrm{NOx}$ levels in SHR, and endogenous $\mathrm{H}_{2} \mathrm{O}_{2}$ upregulates $\mathrm{NO}$ production [27]. The present study reported for the first time that both Ex and tempol increase the levels of plasma and urinary $\mathrm{H}_{2} \mathrm{O}_{2}$ and NOx in SHR, but not in WKY. Since the effects of Ex and tempol are similar, we think that Ex-induced $\mathrm{NO}$ production may also be related to the endogenous $\mathrm{H}_{2} \mathrm{O}_{2}$ in the plasma and urine of SHR. 


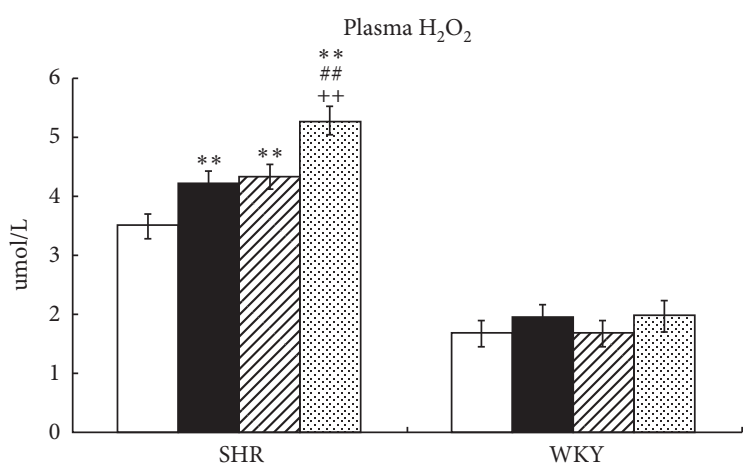

(a)

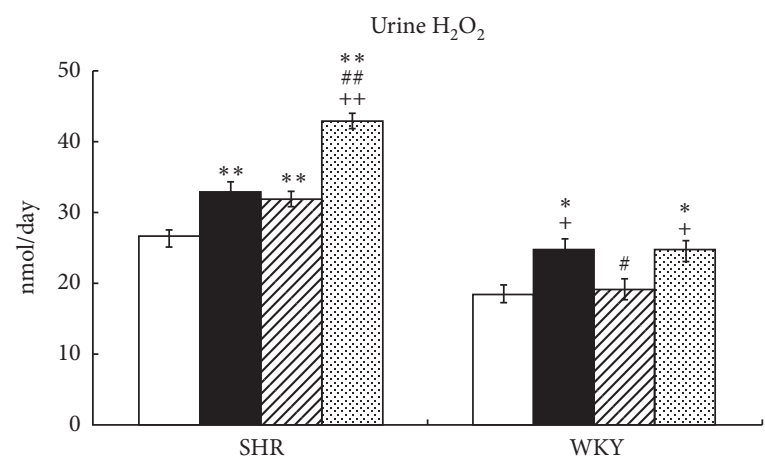

(b)

FIGURE 1: Effects of Ex and tempol on plasma and urinary $\mathrm{H}_{2} \mathrm{O}_{2}$ in SHR and WKY. The $\mathrm{H}_{2} \mathrm{O}_{2}$ in plasma (a) and urine (b) of SHR and WKY was compared among the control group (open bars), Ex group (closed bars), Tmp group (hatched bars), and Ex + Tmp group (checked bars) ( $n=10$ in each group). ${ }^{*} P<0.05$ vs. the control group. ${ }^{* *} P<0.01$ vs. the control group. ${ }^{\# \#} P<0.01$ vs. the Ex group. ${ }^{++} P<0.01$ vs. the Tmp group.

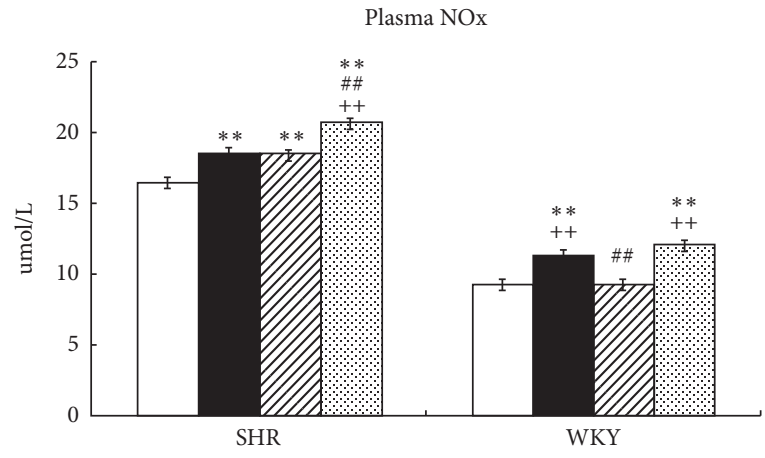

(a)

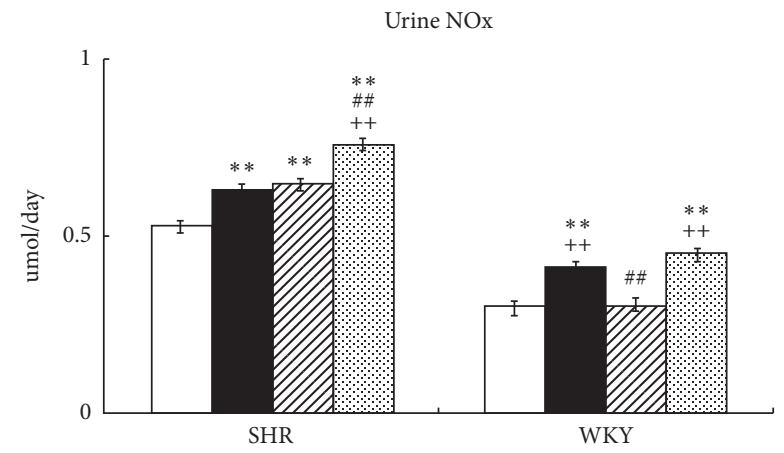

(b)

FIgURE 2: Effects of Ex and tempol on plasma and urinary NOx in SHR and WKY. The NOx in plasma (a) and urine (b) of SHR and WKY was compared among the control group (open bars), Ex group (closed bars), Tmp group (hatched bars), and Ex + Tmp group (checked bars) ( $n=10$ in each group). ${ }^{*} P<0.05$ vs. the control group. ${ }^{* *} P<0.01$ vs. the control group. ${ }^{\# \#} P<0.01$ vs. the Ex group. ${ }^{++} P<0.01$ vs. the Tmp group.

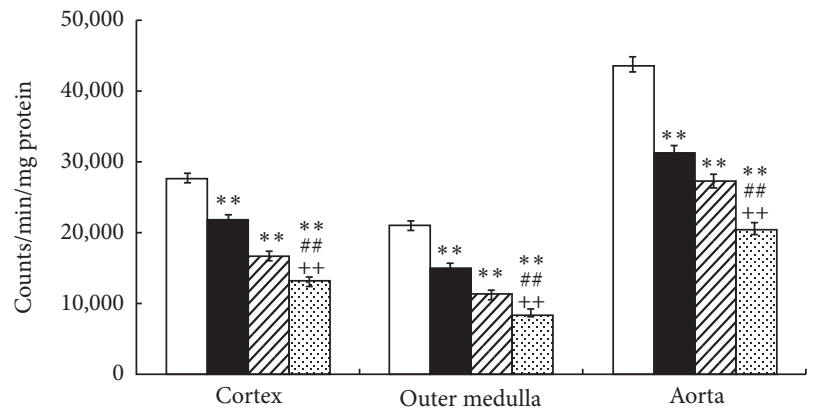

(a)

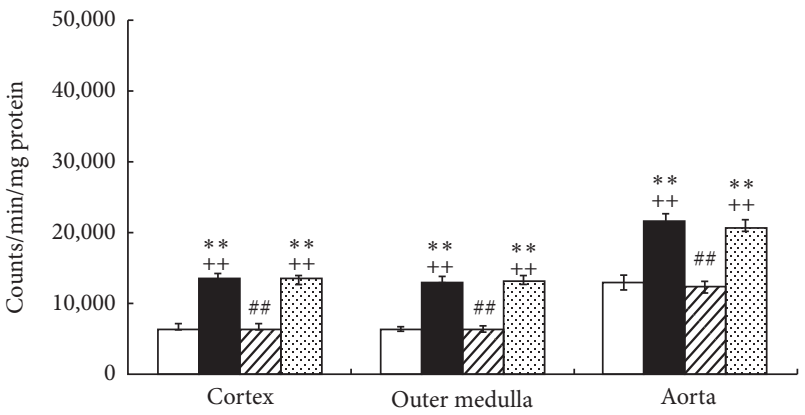

(b)

FIgURE 3: Effects of Ex and tempol on NADPH oxidase activity in SHR and WKY. The NADPH oxidase activity in the renal cortex, outer medulla, and aorta of SHR (a) and WKY (b) was compared among the control group (open bars), Ex group (closed bars), Tmp group (hatched bars), and $\mathrm{Ex}+\mathrm{Tmp}$ group (checked bars) ( $n=10$ in each group). ${ }^{*} P<0.05$ vs. the control group. ${ }^{*} P<0.01$ vs. the control group. ${ }^{\# \#} P<0.01$ vs. the Ex group. ${ }^{++} P<0.01$ vs. the Tmp group.

We have shown that upregulation of $\mathrm{O}_{2}^{-}$in SHR may be dependent on elevated NADPH oxidase activity, and tempol increases the $\mathrm{H}_{2} \mathrm{O}_{2}$ level and decreases NADPH oxidase activity through the activation of SOD in SHR [27]. It has been shown that acute Ex also increases ROS including $\mathrm{H}_{2} \mathrm{O}_{2}$ [36], so we proposed that Ex may also increase the oxidative stress in SHR through a similar mechanism. However, we found that chronic Ex inhibits NADPH oxidase activity but 


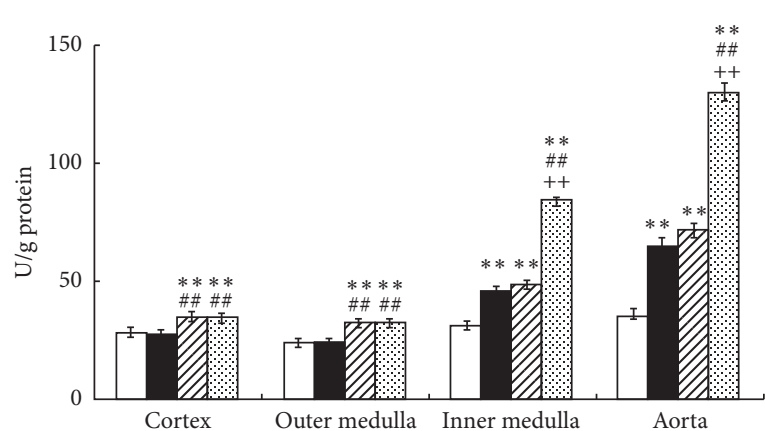

(a)

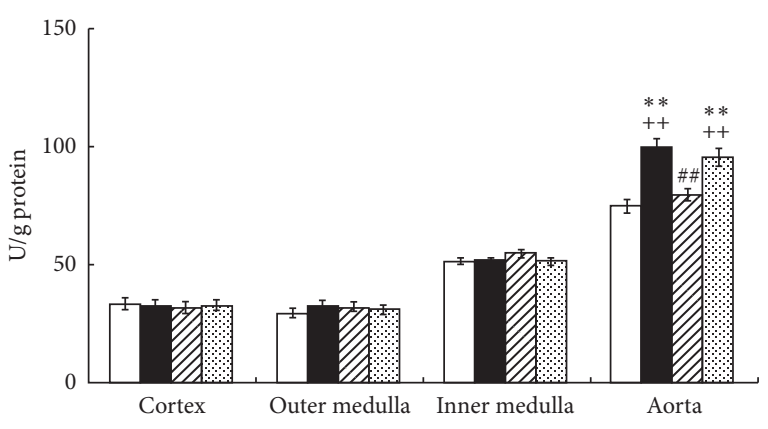

(b)

FIGURE 4: Effects of Ex and tempol on total SOD activity in SHR and WKY. The total SOD activity in the renal cortex, outer medulla, inner medulla, and aorta of SHR (a) and WKY (b) was compared among the control group (open bars), Ex group (closed bars), Tmp group (hatched bars), and Ex + Tmp group (checked bars) ( $n=10$ in each group). ${ }^{*} P<0.05$ vs. the control group. ${ }^{* *} P<0.01$ vs. the control group. ${ }^{\# \#} P<0.01$ vs. the Ex group. ${ }^{++} P<0.01$ vs. the Tmp group.

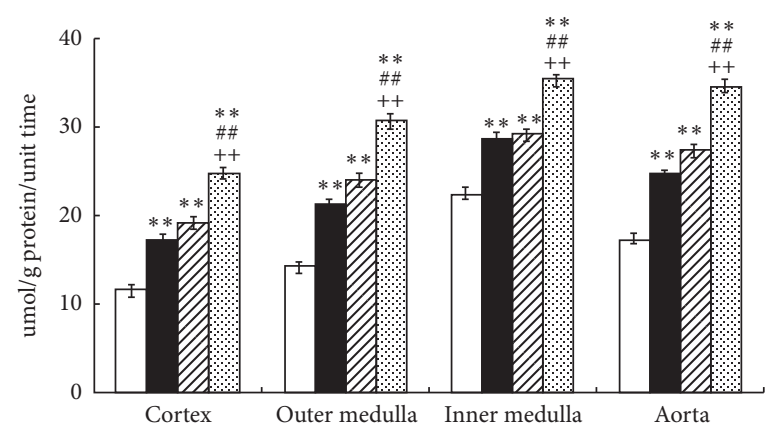

(a)

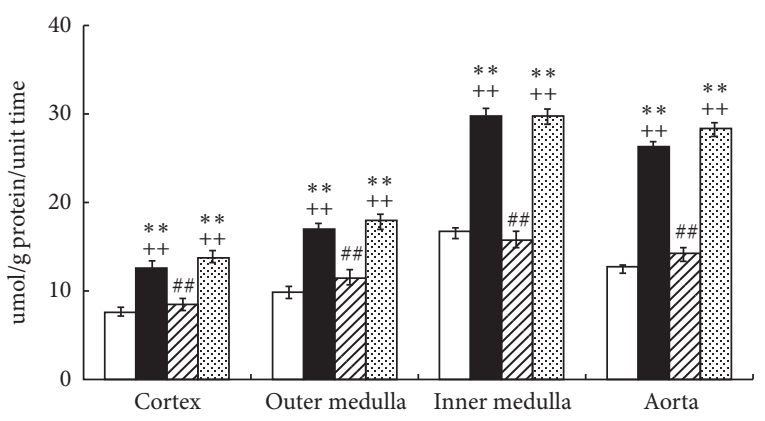

(b)

FIgURE 5: Effects of Ex and tempol on NOS activity in SHR and WKY. The NOS activity in the renal cortex, outer medulla, inner medulla, and aorta of SHR (a) and WKY (b) was compared among the control group (open bars), Ex group (closed bars), Tmp group (hatched bars), and Ex + Tmp group (checked bars) $\left(n=10\right.$ in each group). ${ }^{*} P<0.05$ vs. the control group. ${ }^{* *} P<0.01$ vs. the control group. ${ }^{\# \#} P<0.01$ vs. the Ex group. ${ }^{++} P<0.01$ vs. the Tmp group.

increases SOD activity in SHR. Ito et al. also reported that Ex decreases an index of lipid peroxidation, thiobarbituric acidreactive substances (TBARS) level in the urine of SHR [14]. These data suggest that Ex-induced upregulation of $\mathrm{H}_{2} \mathrm{O}_{2}$ and downregulation of NADPH oxidase activity might depend on increased SOD activity in SHR. Furthermore, we showed an additive effect of the combination of Ex and tempol on $\mathrm{H}_{2} \mathrm{O}_{2}$, NADPH oxidase, and SOD activities in the aorta of SHR. In addition, the previous study has reported that the catalase and glutathione peroxidase activities were not significantly increased in SHR [37]. Subsequently, the metabolism of plasma $\mathrm{H}_{2} \mathrm{O}_{2}$ could not be upregulated. Thus, in the present study, we also reported upregulation of $\mathrm{H}_{2} \mathrm{O}_{2}$ not only in plasma but also in urine of SHR.

It has been shown that Ex has no effect on the plasma and urinary TBARS levels in WKY [14]. In the present study, we demonstrated that Ex increases NADPH oxidase activity both in the kidney and aorta of WKY, but increases SOD activity only in the aorta. Furthermore, Ex-increased $\mathrm{H}_{2} \mathrm{O}_{2}$ is more significant in urine than in plasma of WKY. Taken together, Ex-increased plasma $\mathrm{H}_{2} \mathrm{O}_{2}$ may be carried to the kidney with blood flow and causes the significant increase in urine of WKY. In addition, the degree of Ex-induced
NADPH oxidase activity in the kidney and aorta of WKY was still lower than that in the SHR control, and SOD activity of WKY control is two more times more than of the SHR control. We found that tempol had no effect on SOD activity not only in the WKY control group but also in the Ex-induced WKY group.

We previously reported that both Ex [14] and tempol [27] increase NOS activity and levels of eNOS and nNOS in the kidney and aorta of SHR. In recent studies, exogenous $\mathrm{H}_{2} \mathrm{O}_{2}$ increased the protein level of NOS in the kidney of WKY [27], and exogenous $\mathrm{H}_{2} \mathrm{O}_{2}$ increased eNOS phosphorylation in rats' aortas [36]. A key finding of the present study is the additive effects of Ex and tempol on NOS activity and NOS expression and plasma and urinary $\mathrm{H}_{2} \mathrm{O}_{2}$ levels in SHR. Furthermore, the Ex-increased NO system in the kidney and aorta of WKY is also accompanied with the increase in $\mathrm{H}_{2} \mathrm{O}_{2}$. Thus, endogenous $\mathrm{H}_{2} \mathrm{O}_{2}$ may be a mediator of Ex-induced NOS activity and NOS expression in the kidney and aorta $[29,31]$. The lack of the additive effect of Ex and high dose $(2 \mathrm{mmol} / \mathrm{l})$ tempol on $\mathrm{H}_{2} \mathrm{O}_{2}$ and the $\mathrm{NO}$ system in SHR (data not shown) could be explained by the sufficiency of low dose of tempol on suppressing the oxidative stress in combination with Ex in SHR. 


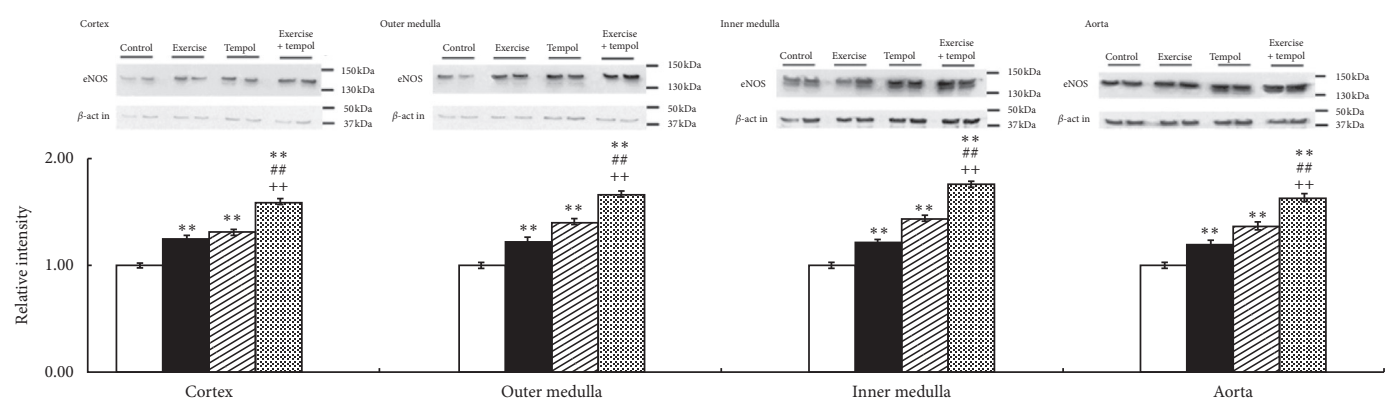

(a)

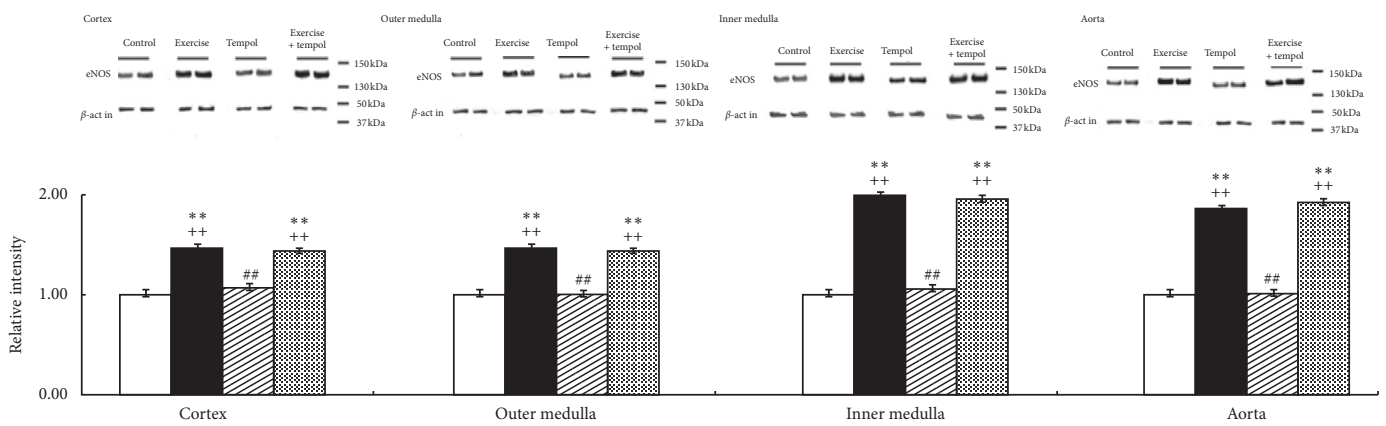

(b)

FIGURE 6: Effects of Ex and tempol on the protein levels of eNOS in SHR and WKY. The protein levels of eNOS in the renal cortex, outer medulla, inner medulla, and aorta of SHR (a) and WKY (b) were compared among the control group (open bars), Ex group (closed bars), Tmp group (hatched bars), and Ex + Tmp group (checked bars) ( $n=10$ in each group). Top panel shows representative immunoblots of eNOS, middle panel shows the immunoblots of $\beta$-actins, and bottom panel shows data of the densitometric analysis. ${ }^{*} P<0.05$ vs. the control group. ${ }^{* *} P<0.01$ vs. the control group. ${ }^{\# \#} P<0.01$ vs. the Ex group. ${ }^{++} P<0.01$ vs. the Tmp group.

Fukai et al. reported that Ex-upregulated the expression levels of vascular eNOS and extracellular SOD in wild-type mice [31]. In the aorta, the Ex-induced blood flow could enhance shear stress, leading to the increase of oxidative stress [12]; in response to the elevated oxidative stress, nuclear factor-erythroid-2-related factor 2 (Nrf2) is synthesized and translocated to the nucleus where it activates the transcription of several antioxidants including SOD [38]. On the other hand, Ex decreased the renal blood flow [39], and shear stress could not increase the SOD activity directly in the kidney. In agreement with these studies, we also found that Ex stimulates the SOD activity in aorta in both SHR and WKY, but not in CO and OM of SHR or the kidney sections of WKY. In the skeletal muscle, Ex also enhanced xanthine oxidase activity [40], resulting in the increase of $\mathrm{O}_{2}{ }^{-}$and SOD activity. Taken together, Ex-increased endogenous $\mathrm{H}_{2} \mathrm{O}_{2}$ in the plasma and urine might be produced in the blood vessel and other organs including the skeletal muscle, but not the kidney. The endogenous $\mathrm{H}_{2} \mathrm{O}_{2}$ was produced by Ex-increased SOD activity and NADPH oxidase activity in the aorta and would be then carried to the kidney with blood flow and causes the increase of the renal NOS activity and expression in SHR and WKY. Further studies are needed to identify the nonrenal $\mathrm{H}_{2} \mathrm{O}_{2}$ mediated mechanism of Ex-induced NOS activity and expression in the kidney.

Ex increased NOS activity and expression in the kidney and aorta, which in turn improved numerous physiological effects of NO in SHR, including vasodilatory responses [41] and various renal effects including regulation of renal hemodynamics and inhibition of renin secretion, tubular $\mathrm{Na}^{+}$reabsorption, tubuloglomerular feedback (TGF), and sympathetic nerve activity $[10,11,42]$. In this regard, we found that both Ex and tempol decrease SBP and increases Ccr, which reflects the glomerular filtration rate (GFR) in SHR, and the effects of the combination of Ex and Tmp on these variables are additive. In consistent with the present results, the recent studies also reported that chronic running Ex upregulates renal NOS expression and normalizes renal NADPH oxidase, accompanied by the improvements of urinary albumin excretion [14], Ccr, glomerulosclerosis, podocyte injury, and tubulointerstitial injury in ZDF rats at an early stage of diabetic nephropathy [43]. It is also reported that tempol increases the GFR, Ccr [24], and the renal medullary blood flow by approximately 50\% in SHR [19]. The renal nephron can be subdivided into at least 13 different segments; the thick ascending limb is not truly a single segment but is at least two segments with cortical and medullary thick ascending limbs that possess quite different characteristics. NO produced by NOS inhibits $\mathrm{NaCl}$ absorption by this segment [44]. Whether the effects of the NO bioavailability in the renal artery and subsequent branches are different need further research and demonstration.

It is widely accepted in recent years that not only $\mathrm{NO}$ but also $\mathrm{H}_{2} \mathrm{O}_{2}$ has the effect on vasodilation. $\mathrm{H}_{2} \mathrm{O}_{2}$ has been shown to be a major component of the endothelium-derived hyperpolarizing factor (EDHF) in several vascular beds in 


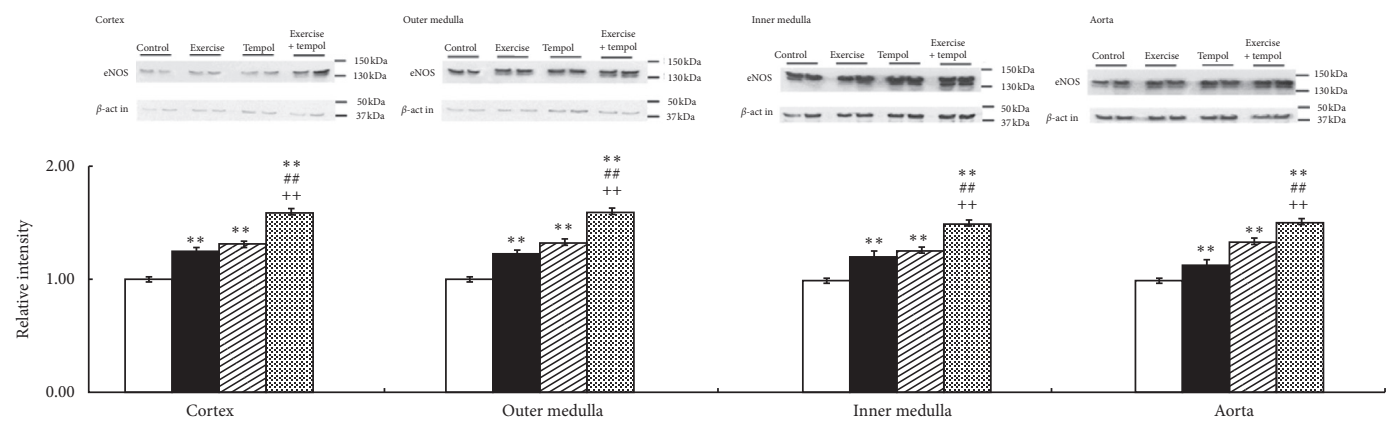

(a)

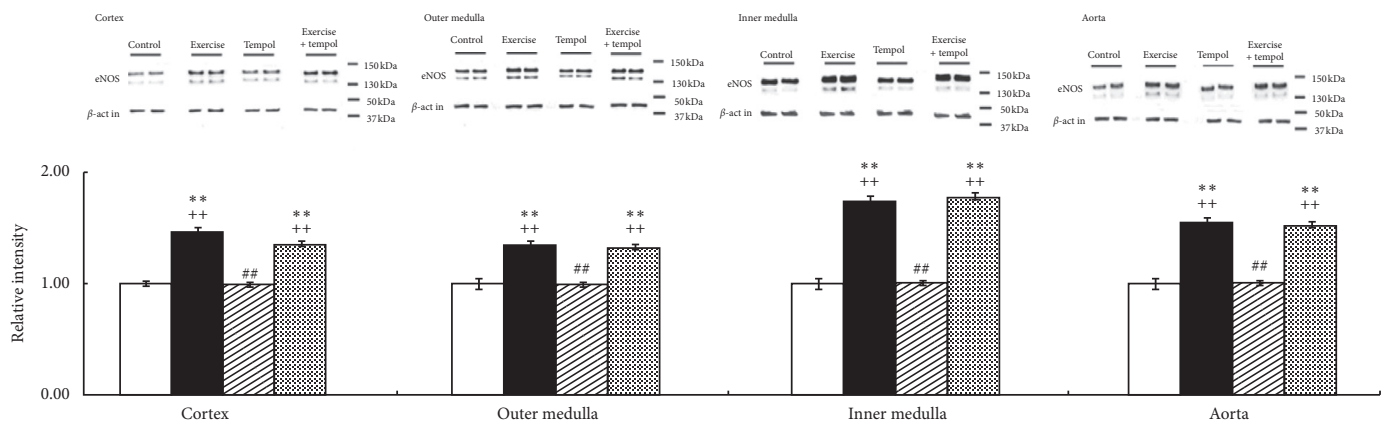

(b)

FIGURE 7: Effects of Ex and tempol on the levels of nNOS in SHR and WKY. The protein levels of nNOS in the renal cortex, outer medulla, inner medulla, and aorta of SHR (a) and WKY (b) were compared among the control group (open bars), Ex group (closed bars), Tmp group (hatched bars), and Ex + Tmp group (checked bars) ( $n=10$ in each group). Top panel shows representative immunoblots of nNOS, middle panel shows the immunoblots of $\beta$-actins, and bottom panel shows data of the densitometric analysis. ${ }^{*} P<0.05$ vs. the control group. ${ }^{* *} P<0.01$ vs. the control group. ${ }^{\# \#} P<0.01$ vs. the Ex group. ${ }^{++} P<0.01$ vs. the Tmp group.

multiple species $[45,46]$, and EDHF is considered to be a major mechanism controlling blood pressure [47]. $\mathrm{H}_{2} \mathrm{O}_{2}$ could also cause interprotein disulfide bond in protein kinase $\mathrm{G}$ (PKG) I- $\alpha$, which activates the kinase independent of the NO-cyclic guanosine monophosphate (cGMP) pathway and coupled to vasodilation [48]. Moreover, pharmacological inhibition of PKG attenuated $\mathrm{H}_{2} \mathrm{O}_{2}$-induced vasorelaxation in mice mesenteries [49]. Consist with the overall results, the antihypertensive and renal protective effects of Ex in SHR might partially depend on the improvement of NO bioactivity, which at least partially rely on the direct effect of $\mathrm{H}_{2} \mathrm{O}_{2}$ on vasorelaxation. However, other studies have yielded conflicting results that $\mathrm{H}_{2} \mathrm{O}_{2}$ revealed vasoconstrictor effects: endothelium-dependent contraction [50] or vasomotor tone $[51,52]$ effects depending on the different vascular beds and the experimental conditions.

In conclusion, the present study indicates that the combination of Ex and Tempol offers additive effects on the NOS activity and levels of eNOS and nNOS in the kidney with decreased renal NADPH oxidase activity and increased arterial SOD activity in SHR. We also demonstrate that the Ex increases the renal NO system with increased renal NADPH oxidase activity and arterial SOD activity in WKY. These results suggest that the Ex-upregulated NO system in the kidney is mediated through the increased endogenous $\mathrm{H}_{2} \mathrm{O}_{2}$ production in the blood vessel and other organs including the skeletal muscle but not the kidney.

\section{Data Availability}

The data used to support the findings of this study are available from the corresponding author upon request.

\section{Conflicts of Interest}

The authors declare that they have no conflicts of interest.

\section{Authors' Contributions}

P.C. and O.I. conceived and designed the study. P.C., D.I., R.R., and Y.Z. performed the experiments and statistical analysis. P.C. wrote the paper. M.K. reviewed and edited the manuscript. All authors read and approved the manuscript.

\section{Acknowledgments}

This work was supported in part by grants from the Ministry of Education, Culture, Sports, Science, and Technology, Japan (nos. 20590694 and 20300184) and the National Natural Science Foundation of China (81301667 and 3A413X793428). The authors thank Zhenwei Gong PhD for the help in editing the manuscript.

\section{References}

[1] L. J. Appel, C. M. Champagne, D. W. Harsha et al., "Effects of comprehensive lifestyle modification on blood pressure 
control: main results of the PREMIER clinical trial," JAMA, vol. 289, no. 16, pp. 2083-2093, 2003.

[2] E. M. Krieger, P. C. Brum, and C. E. Negrão, "State-of-the-art lecture: influence of exercise training on neurogenic control of blood pressure in spontaneously hypertensive rats," Hypertension, vol. 34, no. 4, pp. 720-723, 1999.

[3] K.-D. Schlüter, R. Schreckenberg, and R. M. da Costa Rebelo, "Interaction between exercise and hypertension in spontaneously hypertensive rats: a meta-analysis of experimental studies," Hypertension Research, vol. 33, no. 11, pp. 1155-1161, 2010.

[4] R. E. Shepherd, M. L. Kuehne, K. A. Kenno, J. L. Durstine, T. W. Balon, and J. P. Rapp, "Attenuation of blood pressure increases in Dahl salt-sensitive rats by exercise," Journal of Applied Physiology, vol. 52, no. 6, pp. 1608-1613, 1982.

[5] H. Belabbas, S. Zalvidea, D. Casellas et al., "Contrasting effect of exercise and angiotensin II hypertension on in vivo and in vitro cardiac angiogenesis in rats," American Journal of Physiology-Regulatory, Integrative and Comparative Physiology, vol. 295, no. 5, pp. R1512-R1518, 2008.

[6] M. Kohzuki, M. Kamimoto, X.-M. Wu et al., "Renal protective effects of chronic exercise and antihypertensive therapy in hypertensive rats with chronic renal failure," Journal of $\mathrm{Hy}$ pertension, vol. 19, no. 10, pp. 1877-1882, 2001.

[7] M. Kanazawa, T. Kawamura, L. Li et al., "Combination of exercise and enalapril enhances renoprotective and peripheral effects in rats with renal ablation," American Journal of $\mathrm{Hy}$ pertension, vol. 19, no. 1, pp. 80-86, 2006.

[8] A. Tufescu, M. Kanazawa, A. Ishida et al., "Combination of exercise and losartan enhances renoprotective and peripheral effects in spontaneously type 2 diabetes mellitus rats with nephropathy," Journal of Hypertension, vol. 26, no. 2, pp. 312-321, 2008.

[9] S. Moncada, A. Higgs, and R. Furchgott, "International union of pharmacology nomenclature in nitric oxide research," Pharmacological Reviews, vol. 49, no. 2, pp. 137-142, 1997.

[10] P. F. Mount and D. A. Power, "Nitric oxide in the kidney: functions and regulation of synthesis," Acta Physiologica, vol. 187, no. 4, pp. 433-446, 2006.

[11] P. A. Ortiz and J. L. Garvin, "Role of nitric oxide in the regulation of nephron transport," American Journal of Physiology-Renal Physiology, vol. 282, no. 5, pp. F777-F784, 2002.

[12] R. M. McAllister, S. C. Newcomer, and M. H. Laughlin, "Vascular nitric oxide: effects of exercise training in animals," Applied Physiology, Nutrition, and Metabolism, vol. 33, no. 1, pp. 173-178, 2008.

[13] A. S. Zago and A. Zanesco, "Nitric oxide, cardiovascular disease and physical exercise," Arquivos Brasileiros de Cardiologia, vol. 87, no. 6, pp. e264-e270, 2006.

[14] D. Ito, O. Ito, P. Cao et al., "Effects of exercise training on nitric oxide synthase in the kidney of spontaneously hypertensive rats," Clinical and Experimental Pharmacology and Physiology, vol. 40, no. 2, pp. 74-82, 2013.

[15] Z. Ungvari, A. Csiszar, A. Huang, P. M. Kaminski, M. S. Wolin, and A. Koller, "High pressure induces superoxide production in isolated arteries via protein kinase C-dependent activation of NAD $(\mathrm{P}) \mathrm{H}$ oxidase," Circulation, vol. 108 , no. 10 , pp. 1253-1258, 2003.

[16] S. Adler and H. Huang, "Oxidant stress in kidneys of spontaneously hypertensive rats involves both oxidase overexpression and loss of extracellular superoxide dismutase," American Journal of Physiology-Renal Physiology, vol. 287, no. 5, pp. F907-F913, 2004.
[17] N. Kobayashi, F. A. DeLano, and G. W. Schmid-Schönbein, "Oxidative stress promotes endothelial cell apoptosis and loss of microvessels in the spontaneously hypertensive rats," Arteriosclerosis, Thrombosis, and Vascular Biology, vol. 25, no. 10, pp. 2114-2121, 2005.

[18] L. L. Yanes, D. G. Romero, V. E. Cucchiarelli et al., "Systemic arterial pressure response to two weeks of tempol therapy in SHR: involvement of NO, the RAS, and oxidative stress," American Journal of Physiology-Regulatory, Integrative and Comparative Physiology, vol. 288, no. 1, pp. R229-R233, 2005.

[19] M.-G. Feng, S. A. W. Dukacz, and R. L. Kline, "Selective effect of tempol on renal medullary hemodynamics in spontaneously hypertensive rats," American Journal of PhysiologyRegulatory, Integrative and Comparative Physiology, vol. 281, no. 5, pp. R1420-R1425, 2001.

[20] X. Zhou, H. G. Bohlen, S. J. Miller, and J. L. Unthank, "NAD $(\mathrm{P}) \mathrm{H}$ oxidase-derived peroxide mediates elevated basal and impaired flow-induced NO production in SHR mesenteric arteries in vivo," American Journal of Physiology-Heart and Circulatory Physiology, vol. 295, no. 3, pp. H1008-H1016, 2008.

[21] I. F. Benter, M. H. M. Yousif, G. S. Dhaunsi, J. Kaur, M. C. Chappell, and D. I. Diz, "Angiotensin-(1-7) prevents activation of NADPH oxidase and renal vascular dysfunction in diabetic hypertensive rats," American Journal of Nephrology, vol. 28, no. 1, pp. 25-33, 2008.

[22] Y. Chu, S. Iida, D. D. Lund et al., "Gene transfer of extracellular superoxide dismutase reduces arterial pressure in spontaneously hypertensive rats: role of heparin-binding domain," Circulation Research, vol. 92, no. 4, pp. 461-468, 2003.

[23] C. G. Schnackenberg, W. J. Welch, and C. S. Wilcox, "Normalization of blood pressure and renal vascular resistance in SHR with a membrane-permeable superoxide dismutase mimetic: role of nitric oxide," Hypertension, vol. 32, no. 1, pp. 59-64, 1998.

[24] C. G. Schnackenberg and C. S. Wilcox, "Two-week administration of tempol attenuates both hypertension and renal excretion of 8-Iso prostaglandin F $2 \alpha$," Hypertension, vol. 33, no. 1, pp. 424-428, 1999.

[25] N. D. Vaziri, Z. Ni, and F. Oveisi, "Upregulation of renal and vascular nitric oxide synthase in young spontaneously hypertensive rats," Hypertension, vol. 31, no. 6, pp. 1248-1254, 1998.

[26] N. D. Vaziri, Z. Ni, F. Oveisi, and D. L. Trnavsky-Hobbs, "Effect of antioxidant therapy on blood pressure and NO synthase expression in hypertensive rats," Hypertension, vol. 36, no. 6, pp. 957-964, 2000.

[27] P. Cao, O. Ito, Q. Guo et al., "Endogenous hydrogen peroxide up-regulates the expression of nitric oxide synthase in the kidney of SHR," Journal of Hypertension, vol. 29, no. 6, pp. 1167-1174, 2011.

[28] G. R. Drummond, H. Cai, M. E. Davis, S. Ramasamy, and D. G. Harrison, "Transcriptional and posttranscriptional regulation of endothelial nitric oxide synthase expression by hydrogen peroxide," Circulation Research, vol. 86, no. 3, pp. 347-354, 2000.

[29] N. Lauer, T. Suvorava, U. Ruther et al., "Critical involvement of hydrogen peroxide in exercise-induced up-regulation of endothelial NO synthase," Cardiovascular Research, vol. 65, no. 1, pp. 254-262, 2005.

[30] T. Ziegler, P. Silacci, V. J. Harrison, and D. Hayoz, "Nitric oxide synthase expression in endothelial cells exposed to mechanical forces," Hypertension, vol. 32, no. 2, pp. 351-355, 1998. 
[31] T. Fukai, M. R. Siegfried, M. Ushio-Fukai, Y. Cheng, G. Kojda, and D. G. Harrison, "Regulation of the vascular extracellular superoxide dismutase by nitric oxide and exercise training," Journal of Clinical Investigation, vol. 105, no. 11, pp. 16311639, 2000.

[32] T. Sousa, D. Pinho, M. Morato et al., "Role of superoxide and hydrogen peroxide in hypertension induced by an antagonist of adenosine receptors," European Journal of Pharmacology, vol. 588, no. 2-3, pp. 267-276, 2008.

[33] L. C. Green, D. A. Wagner, J. Glogowski, P. L. Skipper, J. S. Wishnok, and S. R. Tannenbaum, "Analysis of nitrate, nitrite, and $(15 \mathrm{~N})$ nitrate in biological fluids," Analytical Biochemistry, vol. 126, no. 1, pp. 131-138, 1982.

[34] M. M. Bradford, "A rapid and sensitive method for the quantitation of microgram quantities of protein utilizing the principle of protein-dye binding," Analytical Biochemistry, vol. 72, no. 1-2, pp. 248-254, 1976.

[35] D. Ito, O. Ito, N. Mori et al., "Atorvastatin upregulates nitric oxide synthases with Rho-kinase inhibition and Akt activation in the kidney of spontaneously hypertensive rats," Journal of Hypertension, vol. 28, no. 11, pp. 2278-2288, 2010.

[36] V. A. Barbosa, T. F. Luciano, S. O. Marques et al., "Acute exercise induce endothelial nitric oxide synthase phosphorylation via Akt and AMP-activated protein kinase in aorta of rats: role of reactive oxygen species," International Journal of Cardiology, vol. 167, no. 6, pp. 2983-2988, 2013.

[37] C.-D. Zhan, R. K. Sindhu, J. Pang, A. Ehdaie, and N. D. Vaziri, "Superoxide dismutase, catalase and glutathione peroxidase in the spontaneously hypertensive rat kidney," Journal of $\mathrm{Hy}$ pertension, vol. 22, no. 10, pp. 2025-2033, 2004.

[38] S. Ruiz, P. E. Pergola, R. A. Zager, and N. D. Vaziri, “Targeting the transcription factor Nrf2 to ameliorate oxidative stress and inflammation in chronic kidney disease," Kidney International, vol. 83, no. 6, pp. 1029-1041, 2013.

[39] R. M. McAllister, "Adaptations in control of blood flow with training: splanchnic and renal blood flows," Medicine \& Science in Sports \& Exercise, vol. 30, no. 3, pp. 375-381, 1998.

[40] M.-C. Gomez-Cabrera, C. Borrás, F. V. Pallardó, J. Sastre, L. L. Ji, and J. Viña, "Decreasing xanthine oxidase-mediated oxidative stress prevents useful cellular adaptations to exercise in rats," The Journal of Physiology, vol. 567, no. 1, pp. 113-120, 2005.

[41] S. Moncada, "Nitric oxide in the vasculature: physiology and pathophysiology," Annals of the New York Academy of Sciences, vol. 811, no. 1, pp. 60-69, 1997.

[42] J. L. Garvin, M. Herrera, and P. A. Ortiz, "Regulation of renal $\mathrm{NaCl}$ transport by nitric oxide, endothelin, and ATP: clinical implications," Annual Review of Physiology, vol. 73, no. 1, pp. 359-376, 2011.

[43] D. Ito, P. Cao, T. Kakihana et al., "Chronic running exercise alleviates early progression of nephropathy with upregulation of nitric oxide synthases and suppression of glycation in zucker diabetic rats," PLoS One, vol. 10, no. 9, Article ID e0138037, 2015.

[44] G. V. Agustin, D. Pablo, and L. G. Jeffrey, "Resveratrol increases nitric oxide production in the rat thick ascending limb via $\mathrm{Ca}^{2+}$ /calmodulin," PLoS One, vol. 9, no. 10, Article ID e110487, 2014.

[45] H. Shimokawa and K. Morikawa, "Hydrogen peroxide is an endothelium-derived hyperpolarizing factor in animals and humans," Journal of Molecular and Cellular Cardiology, vol. 39, no. 5, pp. 725-732, 2005.
[46] H. Shimokawa, "Hydrogen peroxide as an endothelium-derived hyperpolarizing factor," Pflügers Archiv-European Journal of Physiology, vol. 459, no. 6, pp. 915-922, 2010.

[47] G. Edwards, M. Félétou, and A. H. Weston, "Endotheliumderived hyperpolarising factors and associated pathways: a synopsis," Pflügers Archiv-European Journal of Physiology, vol. 459, no. 6, pp. 863-879, 2010.

[48] J. R. Burgoyne, M. Madhani, F. Cuello et al., "Cysteine redox sensor in PKGIa enables oxidant-induced activation," Science, vol. 317, no. 5843, pp. 1393-1397, 2007.

[49] O. Prysyazhna, O. Rudyk, and P. Eaton, "Single atom substitution in mouse protein kinase $G$ eliminates oxidant sensing to cause hypertension," Nature Medicine, vol. 18, no. 2, pp. 286-290, 2012.

[50] Y.-J. Gao and R. M. K. W. Lee, "Hydrogen peroxide is an endothelium-dependent contracting factor in rat renal artery," British Journal of Pharmacology, vol. 146, no. 8, pp. 1061-1068, 2005.

[51] Y. J. Gao and R. M. K. W. Lee, "Hydrogen peroxide induces a greater contraction in mesenteric arteries of spontaneously hypertensive rats through thromboxane A2 production," British Journal of Pharmacology, vol. 134, no. 8, pp. 16391646, 2001.

[52] J. Z. Shen, X. F. Zheng, E. Q. Wei, and C. Y. Kwan, "Evidence against inhibition of sarcoplasmic reticulum $\mathrm{Ca}^{2+}$-pump as mechanism of $\mathrm{H}_{2} \mathrm{O}_{2}$-induced contraction of rat aorta," Acta Pharmacologica Sinica, vol. 22, no. 6, pp. 498-504, 2001. 